\title{
Erratum to: Elevated homocysteine level in first-episode schizophrenia patients - the relevance of family history of schizophrenia and lifetime diagnosis of cannabis abuse
}

\author{
Blazej Misiak • Dorota Frydecka • Ryszard Slezak • \\ Patryk Piotrowski $\cdot$ Andrzej Kiejna
}

Published online: 22 May 2014

(C) Springer Science+Business Media New York 2014

Erratum to: Metab Brain Dis (2014)

DOI 10.1007/s11011-014-9534-3

The original version of this article unfortunately contained a mistake. Treatment duration should refer to first-episode schizophrenia patients as it was provided in the last version of article submitted to the journal, not to healthy controls. The correct Table 2 is presented here.

Table 2 Correlation coefficients for confounders associated with antipsychotic treatment and cigarette smoking $(p>0.05)$

\begin{tabular}{|c|c|c|c|c|c|c|}
\hline & \multicolumn{4}{|l|}{ FES patients } & \multicolumn{2}{|l|}{$\mathrm{HC}$} \\
\hline & Pack-year index & Fagerström score & Chlorpromazine equivalent & Treatment duration & Pack-year index & Fagerström score \\
\hline Glucose & $r=-0.070$ & $r=-0.078$ & $r=-0.058$ & $r=-0.048$ & $r=0.025$ & $r=0.023$ \\
\hline LDL & $r=0.195$ & $r=0.145$ & $r=0.122$ & $r=-0.034$ & $r=0.243$ & $r=0.237$ \\
\hline HDL & $r=-0.090$ & $r=-0.101$ & $r=-0.143$ & $r=-0.100$ & $r=-0.007$ & $r=-0.010$ \\
\hline $\mathrm{TC}$ & $r=0.180$ & $r=0.125$ & $r=0.023$ & $r=-0.094$ & $r=0.184$ & $r=0.186$ \\
\hline TG & $r=0.004$ & $r=-0.016$ & $r=0.045$ & $r=-0.164$ & $r=0.094$ & $r=0.105$ \\
\hline Hcy & $r=0.058$ & $r=0.062$ & $r=-0.049$ & $r=-0.136$ & $r=0.037$ & $r=0.040$ \\
\hline Folate & $r=-0.134$ & $r=-0.168$ & $r=-0.045$ & $r=0.099$ & $r=0.065$ & $r=0.073$ \\
\hline Vitamin B12 & $r=0.006$ & $r=0.028$ & $r=0.064$ & $r=0.189$ & $r=-0.083$ & $r=-0.062$ \\
\hline
\end{tabular}

Abbreviations: BMI body mass index, FES first-episode schizophrenia, $H C$ healthy controls, $H c y$ homocysteine, $H D L$ high density lipoproteins, HyperHcy hyperhomocysteinemia, $L D L$ low density lipoproteins, $T C$ total cholesterol, $T G$ triglycerides

The online version of the original article can be found at http://dx.doi.org/ 10.1007/s11011-014-9534-3.

B. Misiak $(\varangle) \cdot$ D. Frydecka $\cdot$ P. Piotrowski $\cdot$ A. Kiejna Department of Psychiatry, Wroclaw Medical University,

10 Pasteur Street, 50-367 Wroclaw, Poland

e-mail:mblazej@interia.eu

B. Misiak $\cdot$ R. Slezak

Department of Genetics, Wroclaw Medical University,

1 Marcinkowski Street, 50-368 Wroclaw, Poland 